\title{
Implementando Estrategias de mercado ¿Gestión estratégica de marketing o una combinación?
}

\section{Implementing Marketing Strategies ¿Strategic marketing management or a com- bination?}

\author{
Omaira C. Martínez-Moreno* \\ Universidad Autónoma de Baja California - México \\ ORCID iD: https://orcid.org/0000-0002-9562-1840 \\ omairam@uabc.edu.mx
}

\author{
Yannet Hernández-Ibarra \\ Universidad Autónoma de Baja California - México \\ ORCID iD: https://orcid.org/0000-0002-9767-3388 \\ yannet.hernandez@uabc.edu.mx \\ Juan C. Sandoval-Zavala \\ Universidad Autónoma de Baja California - México \\ ORCID iD: https://orcid.org/0000-0002-7960-9147 \\ juan.carlos.sandoval.zavala@uabc.edu.mx
}

* Autor a quien debe ser dirigida la correspondencia
Fecha de recepción: 14/08/2021

Fecha de evaluación: 27/08/2021

Fecha de aceptación: 14/10/2021

Cómo citar: Martínez-Moreno, O., Hernández-Ibarra, Y., \& Sandoval-Zavala, J. (2021). Implementando Estrategias de mercado ¿Gestión estratégica de marketing o una combinación?. Revista Cientifica Anfibios, 4(2), 20-28. https://doi.org/10.37979/afb.2021v4n2.91

\section{Resumen}

Con los requerimientos del entorno empresarial de ajustarse a las necesidades de sus clientes, resulta imprescindible implementar las estrategias de marketing adecuadas para llegar al público objetivo, sin embargo, esta estrategia puede que requiera esfuerzos de otras dependencias de la organización. Es así que se pretende examinar la relación entre gestión estratégica y marketing en aspectos primordiales como el interfaz entre cuestiones estratégicas y cuestiones de marketing y cómo se gestiona en entornos prácticos, Esta propuesta va dirigida a ejecutivos de empresas, gerentes y académicos de marketing. Para tales fines se plantea una revisión de la literatura en esta temática. Los resultados que contienen están respaldados por información empírica acerca de las perspectivas convencionales sobre el contenido de marketing, gestión estratégica y toma de decisiones, también se discuten los cambios organizativos y los argumentos teóricos que han socavado en estos conceptos.

\section{Palabras clave}

Gestión estratégica; gestión del marketing; clientes; implementación

\begin{abstract}
With the requirements of the business environment to adjust to the needs of their customers, it is essential to implement appropriate marketing strategies to reach the target audience, however, this strategy may require efforts from other organizational units. Thus, we intend to examine the relationship between strategic management and marketing in key aspects such as the interface between strategic issues and marketing issues and how it is managed in practical settings. To this end, a review of the literature on this subject is proposed. The findings are supported by empirical information about conventional perspectives on marketing content, strategic management and decision making, as well as organizational changes and theoretical arguments that have undermined these concepts.
\end{abstract}

\section{Keywords}

Strategic management; marketing management; customers; implementation 


\section{Introducción}

La gestión estratégica y el marketing muchas veces suelen dividirse en las organizaciones (Y.S. Chen \& Chen, 2009; Larke et al., 2018; Madden et al., 2006; Park et al., 2010), para Morris y Pit (1992) la división de estos dos ocasiona problemas en el desarrollo de prácticas organizacionales efectivas en el contexto de cambios en el entorno, la estructura de las corporaciones y la construcción de cursos de administración que son relevantes para la situación practica cambiante.

La gestión estratégica se entiende según Schendel y Hofer (1979) como "Un proceso que se ocupa del trabajo empresarial organizacional, la renovación y el crecimiento organizacional, especialmente involucrando la formulación y uso de estrategias que guíen las operaciones de la organización. Por otro lado, el marketing es concebido como un conjunto de estrategias y técnicas que se enfocan en el posicionamiento del mercado, en mercadeo de productos y una ventaja competitiva sostenible (Greenley \& Aaby, 1992).

Por lo anterior Day (1992) resume la diferencia entre estas dos áreas asociando que la planificación estratégica consiste en alinear el negocio con el entorno esperado, y el marketing siempre ha sido una función límite entre la empresa y sus clientes internos como elementos estratégicos que impulsan la entrega de valor a los clientes externos, mediante canales estratégicamente establecidos y siempre con una visión hacia el entorno (Arbeláez et al., 2017). Esta postura nos deja claro los roles y funciones de gestión estratégica y marketing.

No obstante, se requiere de un estudio que profundice estas diferencias y semejanzas, permitiendo a los gerentes de las organizaciones dilucidar este contraste de tal manera que puedan tomar decisiones acordes con el entorno organizacional en donde se encuentran. Por eso se propone un estudio que posibilite verificar la relación entre la gestión estratégica y el marketing en aspectos clave, como la interfaz entre los problemas estratégicos y los problemas de marketing y cómo gestionarlos en el entorno real.

Para tales fines se parte de referentes teóricos con la finalidad de profundizar tanto la conceptualización, las diferencias y semejanzas de estos dos conceptos tan fundamentales para el ámbito empresarial en la actualidad, posteriormente se plantean los aspectos metodológicos enmarcados en una revisión de la literatura para dar paso a los resultados demostrando que la gestión estratégica y el marketing deben ir de la mano y coordinados con la finalidad de trazar una senada de crecimiento positiva para las organizaciones en la actualidad.

\section{Fundamentos Teóricos}

De acuerdo con la Junta de Normas de Responsabilidad de Marketing (MASB), la definición formal de gestión de marketing es: «El proceso de establecer objetivos de marketing para una organización (teniendo en cuenta los recursos internos y las oportunidades de mercado), la planeación y ejecución de actividades para cumplir estos objetivos, y la medición del progreso hacia el logro de estos»».

Los dos objetivos principales de la gestión de marketing son maximizar la cuota de mercado de la empresa dentro de la industria en general, así como la satisfacción del cliente de la base de consumidores dentro de su cuota de mercado ya capturada (Halim et al., 2019; Matthyssens, 2019; Sainidis et al., 2019; Vieira et al., 2019). En pocas palabras, se trata de gestionar todo lo que se necesita para impulsar la mercadotecnia y las ventas en el negocio (Mullins \& Panagopoulos, 2019; Pozo \& Tachizawa, 2018; Sukier et al., 2018; Vieira et al., 2019). Esto significa crear, ejecutar y medir un plan para que la empresa alcance su mercado objetivo, se comunique con este y lo convierta.

En la actualidad, muchas industrias están viviendo momentos difíciles que obligan a los ejecutivos a simplificar, achicarse y asegurarse de que la organización sea lo más eficiente posible(Bachrach et al., 2017; Milichovsky \& Simberova, 2015; Ramanathan et al., 2016). ¿Por qué la gestión de marketing debe seguir siendo una prioridad, incluso cuando la economía se dirige hacia una posible recesión?

Tal como lo dijo Harvard Business Review durante la recesión anterior: "Durante las recesiones es más importante que nunca recordar que los clientes leales son la fuente principal y permanente del flujo de fondos y crecimiento orgánico. El marketing no es opcional: es un «costo positivo», 
esencial para generar ingresos de estos clientes clave y de otros". A partir de este breve contexto económico se abordan los principales ejes conceptuales.

\section{Estrategia de mercado}

Una estrategia de mercadeo es la creación de acciones o tácticas que lleven al objetivo fundamental de incrementar las ventas y lograr una ventaja competitiva sostenible, la estrategia incluye todo tipo de actividades básicas, de largo como de corto plazo, así como actividades que tienen que ver con el análisis de la situación estratégica inicial de una compañía (Y.-S. Chen \& Chen, 2009; Larke et al., 2018; Nowlin et al., 2018; Rojas De Francisco et al., 2016).

Según los autores clásicos (Philip Kotler, Gary Armstrong), los engranajes que conforman la estrategia de marketing son las ya conocidas 4 "P"; Producto: Entendido como conjunto de atributos que las organizaciones ofrecen a sus clientes, ya sea en forma de bien o servicio, para generarles valor y satisfacción (Marin Carrillo et al., 2004; Weiss et al., 2015). Precio: Entendido como todo esfuerzo que el cliente lleva a cabo para obtener el producto ofrecido. Incluye el costo monetario, el costo de oportunidad, el tiempo y el esfuerzo psicológico (Z. Chen \& Dubinsky, 2003; Halim et al., 2019; Madden et al., 2006). Promoción o comunicación: Entendido como modelo de interacción entre la organización y sus clientes para comunicar los beneficios del producto y recibir retroalimentación por parte de éstos últimos $(\mathrm{Pa}-$ tiño et al., 2018; Sam, 2017). Plaza o distribución: Entendido como el conjunto de canales o medio por los cuales viajan la información y los productos tangibles desde la organización hacia el cliente y viceversa (Ramirez Angulo et al., 2013; Reddy \& Reinartz, 2017).

Sin embargo, hay tres elementos fundamentales que se desprenden de los anteriores y es necesario destacarlos. Ellos son, segmentación conceptualizado como una forma en que la organización clasifica y agrupa a sus clientes según algún criterio lógico, para ofrecer productos que se adapten en un mayor grado a las necesidades de cada segmento (Boone \& Roehm, 2002; Kumar \& RRK, 2017); Posicionamiento asociado con un modo en que la organización busca ser percibida tanto por el cliente como por la so- ciedad en general y otros grupos relacionados (proveedores, gobierno, distribuidores, competidores, etc.). Para ello es necesario fijar la visión, misión, UENs y objetivos estratégicos (Larke et al., 2018; Milichovsky \& Simberova, 2015) y finalmente la calidad como un el elemento secreto de la receta para el éxito. No debe entenderse como la ausencia de errores o fallas, sino como una cultura que permita mejorar (Alence, 2004; Barton et al., 2008; Hu et al., 2009; Martínez \& Martínez, 2009)

\section{Gestión estratégica}

Las estrategias de gestión se asocian con la administración la gestión de los planes de acción y objetivos definidos para el éxito de una empresa. En la gestión estratégica, se preparan proyectos y cambios estratégicos, además de monitorear y gestionar lo que se ha implementado (Castellanos Narciso \& Pulido, 2014; Madden et al., 2006; Sikora et al., 2016). Es una forma de optimizar y hacer más efectiva la gestión de un negocio y, como se desprende del propio nombre, esta forma de gestión existe para gestionar y dirigir un negocio siguiendo estrategias que pasan por toda la estructura de la organización (Castellanos Narciso \& Pulido, 2014; Fraj et al., 2015; Qazi et al., 2019; Vesga R., 2013). En resumen, la gerencia estratégica es responsable de la ejecución de lo que fue establecido en el planeamiento estratégico.

En palabras de Betancourt (2007), la gestión estratégica puede ser vista como "el arte y/o ciencia de anticipar y gerenciar participativamente el cambio con el propósito de crear permanentemente estrategias que permitan garantizar el futuro de la organización" (p. 106).

Es a través de la gestión estratégica que haces que los profesionales que trabajan en la empresa tomen conciencia de sus responsabilidades (Naranjo-Africano, G., \& Giraldo, 2020; Wu et al., 2018). Esto refleja el aumento de la productividad y, en consecuencia, de la competitividad del negocio, y también ayuda en el uso adecuado de los recursos, la reducción de los residuos y la maximización de la rentabilidad de cada acción desencadenada por la empresa (Covin et al., 2006; D’Allura, 2019; Kowalkowski et al., 2017). Por lo tanto, es en la gestión estratégica que debes 
ser guiado para promover la mejora continua de tu negocio, además de asegurar la supervivencia de la empresa en el tiempo

\section{Metodología}

Con base en una investigación cualitativa, comparativa y descriptiva, se plantea una revisión de la literatura, con el propósito de examinar la relación entre la gestión estratégica y el marketing en ámbitos fundamentales, como la interfaz entre los aspectos estratégicos y los aspectos del marketing y cómo gestionar en el medio ambiente. Para tales fines se buscaron publicaciones de corte científico en bases de datos reconocidas como Scopus y Web Of Science (WOS). Los criterios de inclusión fueron que la ubicación estuviera directamente relacionada con el la temática en cuestión además de ser los registros con mayor citación en la literatura académica, esto con la finalidad de elegir aquellas publicaciones de mayor impacto académico. Posteriormente se analizaron de forma comparativa los resultados de los documentos y a partir de allí se generaron resultados y conclusiones fundamentadas en trabajos investigativos teóricos y empíricos.

\section{Resultados}

La visión de la gestión estratégica se enfoca más por las decisiones tomadas de los altos mandos de una organización y se preocupa por las elecciones del rumbo que tomara la empresa sobre el negocio y el despliegue de recursos de la cartera del negocio. Varadarajan (1992) enlista los temas que se consideran centrales para esta visión de la gestión estratégica. En primera instancia la incorporación de nuevos negocios al portafolio de la firma, es decir, la diversificación de los productos y servicios (Bhattacharyya \& Bose, 2020). En segunda instancia la eliminación de negocios de la cartera de la empresa o también denominada desinversión (Molina-Morales et al., 2011). En tercer lugar la gestión eficaz de los negocios retenidos, esto implica un análisis de cartera de productos, asignación de misiones y asignación de recursos (Aragón Sánchez \& Bañón Rubio, 2006) y por ultimo aumentar o disminuir el alcance de las etapas de valor agregado en las que participan los negocios individuales estos una integración o contracción verticales (Larke et al., 2018).
La visión del marketing recae en cuatro áreas principales de pensamiento y desarrollo. En primer lugar, el marketing se considera una filosofía organizativa o de gestión en términos de logros de la orientación al mercado, "el marketing es una forma de hacer negocios", en segundo lugar el marketing se ve como un conjunto de cuestiones estratégicas que buscan el posicionamiento de mercado, establecer un mercado de productos y una ventaja competitiva, en tercer lugar, el marketing se encarga de la gestión de políticas de productos, precios, comunicaciones y canales de distribución y por último el cuarto lugar el marketing se ve más como una función de procesamiento de información, que interpreta el entorno del mercado para la organización. (Greenley y Aaby, 1992; Kotler, 1996; McKenna, 1991).

\section{La interfaz confusa marketing-estrategia}

Dada la superposición conceptual y practica cada vez más evidente entre el marketing y la gestión estratégica, entonces surge la pregunta ¿por qué los académicos y los formadores deberían persistir en la enseñanza de las áreas por separado?

Existen tres razones por la cuales no parece viable la unión entre las dos áreas. En primer lugar, es necesario considerar como se relacionan las cuestiones estratégicas del marketing con la estrategia corporativa, segundo, es probable que cualquier análisis más profundo sugiera que, de hecho, existe cierta competencia entre los conceptos de marketing y estrategia y tercero, ambas están experimentado desafíos generados por los desarrollos en las formas y prácticas corporativas y cambios en el entorno (Piercy, Harris, Peters y Lane, 1997).

Para muchos ejecutivos importa poco si algo es cuestión de "marketing" o cuestión de "gestión estratégica", siempre y cuando el problema se aborde y se resuelva, es bien conocido que el mundo de los negocios gira entorno a buscar resultados y obtener rendimientos.

Según el análisis de (Piercy et al., 1997) las razones por las cuales las perspectivas convencionales de separar el marketing y la gestión estratégica se centran en las siguientes cuestiones: ¿por qué mover el barco? Síndrome: el mantenimiento y el desarrollo incremental de la separación tra- 
dicional entre marketing y gestión estratégica es sencillamente más fácil para los académicos y los formadores. Adicionalmente algunos académicos pueden enseñar a los paradigmas convencionales porque creen que, sobre bases genuinas, racionales y prescriptivas, así es como las organizaciones "deberían" abordar estos problemas y que esto debería reflejarse en la forma en que se educan los gerentes. La división tradicional se mantiene porque la práctica dominante está en las escuelas de negocios de EE.UU.

Por su parte, el síndrome de "no sabemos cómo, así que lo ignoramos" es la sugerencia de que las divisiones convencionales de "temas" existen porque se carece de un mecanismo acordado para desarrollar alternativas de manejar estas áreas y relacionarlas juntas.
Claramente existe una resistencia por parte de algunos académicos, influidos por la creencia que existen términos disciplinarios inequívocos entre las disciplinas comerciales, sin embargo, el autor del artículo destaca la posibilidad de brindar alguna asistencia para desarrollar un enfoque alternativo para el diseño y mantenimiento de la interfaz de gestión estratégica y marketing.

\section{Un marco para reflexionar el marketing y la gestión estratégica}

Se propone un marco basado en un mecanismo simple para identificar y clasificar las diferentes áreas de estudio y análisis de ambos temas de acuerdo con Piercy et al., (1997), en la siguiente ilustración

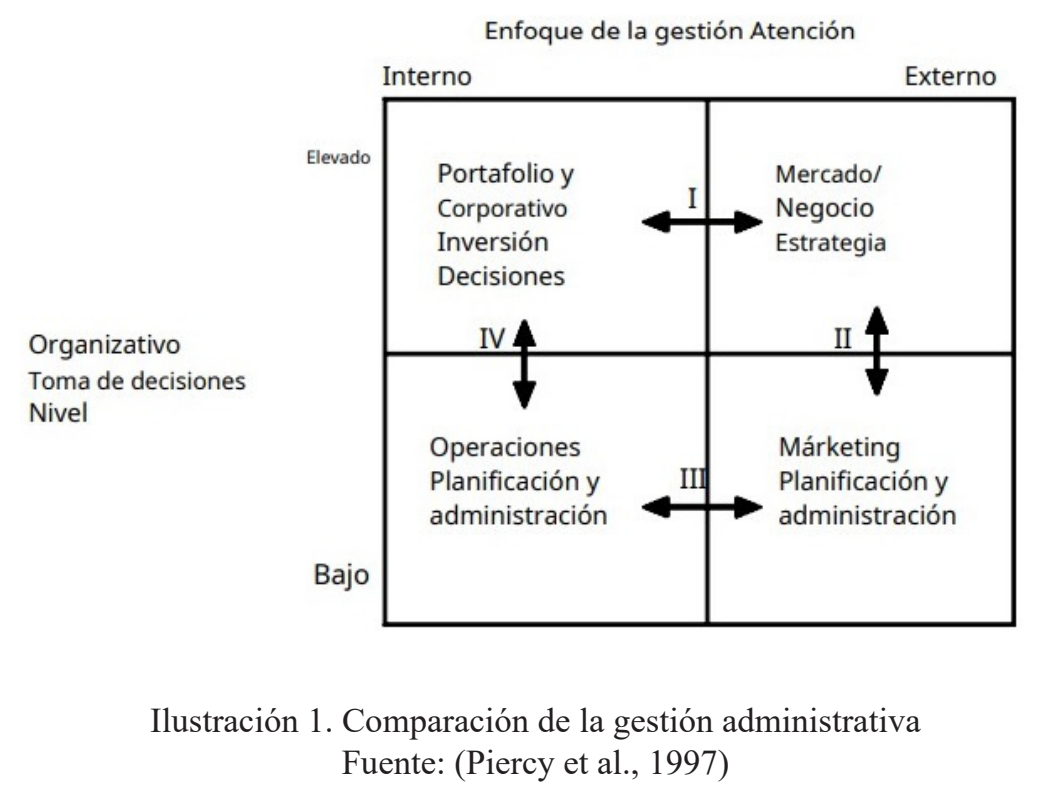

El modelo utiliza dos criterios de clasificación: la toma de decisiones organizativas y el enfoque de atención de la dirección. La aplicación de estos ejes sugiere cuatro áreas interrelacionadas de análisis, estudio y toma de decisiones, estas cuatro áreas son las siguientes.

La decisión de inversión corporativa y de cartera: esta área se enfoca en los problemas internos, en gestionar los modelos de cartera y financieros, reconoce que la importancia de la asignación y diversificación de recurso podría construir carteras efectivas.

Mercado=estrategia comercial: El objetivo de esta área son la ventaja competitiva y la estrategia (SBU), las herramientas adecua- das para este enfoque incluyen análisis DAFO, análisis estructural de la industria, identificación de grupos estratégicos y evaluación de la posición empresarial.

Planificación y gestión de operaciones: el enfoque principal de este rubro es la identificación, aplicación y análisis de técnicas apropiadas para evaluar las especializaciones del negocio a nivel operativo y la identificación y explotación de la competencia central de la organización.

Planificación y gestión de marketing: esta área se centra en los aspectos técnicos y profesionales de la gestión de programas de marketing y ventas a nivel operativo. 


\section{Conclusión}

El articulo propone un marco que clasifica los problemas que enfrenta la interrelación de marketing y la gestión estratégica, se destaca la importancia de relacionar las dos áreas para trabajar de una manera más eficiente.

El articulo resalta la diferencia de pensamiento entre los ejecutivos empresariales y los académicos sobre la interfaz de marketing y gestión estratégica, por un lado, los ejecutivos se centran en resultados mas que en los medios a ellos les da lo mismo si es cuestión de marketing o es cuestión de gestión estratégica, mientras que a los académicos le interesa sustentas los métodos y procesos que ayudan a llegar hacia el objetivo.

En lo personal y con el análisis del articulo considero que el problema no es si separamos o juntamos las dos áreas, sino como lo dice el autor, el problema real es que como se desconoce el método por el cual las dos áreas trabajarían de manera eficiente entonces se decide ignorar el tema, por ello la importancia de seguir estudiando y desarrollar estos modelos que contribuyen a la mejora continua.

\section{Referencias}

Alence, R. (2004). Political institutions and developmental governance in sub-Saharan Africa. Journal of Modern African Studies, 42(2), 163-187. https://doi.org/10.1017/S0022278X04000084

Aragón Sánchez, A., \& Bañón Rubio, A. (2006). Factores explicativos del éxito competitivo: el caso de las PyMEs del estado de Veracruz. Contaduría y Administración, 216(June), 35-69.

Arbelaez, D. C., Castillo, E. M. E., \& Licona, J. M. (2017). Endomarketing. Revista Cultural Unilibre, (2), 50-57.

Bachrach, D. G., Mullins, R. R., \& Rapp, A. A. (2017). Intangible sales team resources: Investing in team social capital and transactive memory for market-driven behaviors, norms and performance. Industrial Marketing Management, 62, 88-99. https://doi.org/10.1016/j.indmarman.2016.08.001

Barton, G. R., Sach, T. H., Doherty, M., Avery, A. J., Jenkinson, C., \& Muir, K. R. (2008). An assessment of the discriminative ability of the EQ-5Dindex, SF-6D, and EQ VAS, using sociodemographic factors and clinical conditions. European Journal of Health Economics, 9(3), 237-249. https://doi.org/10.1007/s10198-007-0068-z

Bhattacharyya, S., \& Bose, I. (2020). S-commerce: Influence of Facebook likes on purchases and recommendations on a linked e-commerce site. Decision Support Systems, 138(January), 113383. https://doi.org/10.1016/j.dss.2020.113383

Boone, D. S., \& Roehm, M. (2002). Evaluating the Appropriateness of Market Segmentation Solutions Using Artificial Neural Networks and the Membership Clustering Criterion. Marketing Letters, 13(4), 317-333. https://doi.org/10.1023/A:1020321132568

Castellanos Narciso, J. E., \& Pulido, M. A. C. (2014). Una Mirada a la Evolución Histórica de la Estrategia Organizacional. Reivista Estudios Avanzados de Liderazgo, 1(3), 1-24.

Chen, Y.-S., \& Chen, C.-C. (2009). A model of factors moderating the relationship between new product development and company performance. Social Behavior and Personality: An International Journal, 37(8), 1043-1049. https://doi.org/10.2224/sbp.2009.37.8.1043

Chen, Z., \& Dubinsky, A. J. (2003). A Conceptual Model of Perceived Customer Value in E-Commerce: A Preliminary Investigation. Psychology and Marketing, 20(4), 323-347. https://doi. org/10.1002/mar.10076 
Covin, J. G., Green, K. M., \& Slevin, D. P. (2006). Strategic process effects on the entrepreneurial orientation-sales growth rate relationship. Academy of Management 2005 Annual Meeting: A New Vision of Management in the 21st Century, AOM 2005, 30(1), 57-81. https://doi. org/10.5465/ambpp.2005.18778648

D'Allura, G. M. (2019). The leading role of the top management team in understanding family firms: Past research and future directions. Journal of Family Business Strategy, 10(2), 87-104. https://doi.org/10.1016/j.jfbs.2018.12.001

Fernando G. (2011). Análisis de la ultima tendencia en el mercado de consumo. Obtenido de. https:// bdigital.uncu.edu.ar/objetos_digitales/4868/gallardocetrabajodeinvestigacion.pdf

Fraj, E., Matute, J., \& Melero, I. (2015). Environmental strategies and organizational competitiveness in the hotel industry: The role of learning and innovation as determinants of environmental success. Tourism Management, 46, 30-42. https://doi.org/10.1016/j.tourman.2014.05.009

Halim, K. K., Halim, S., \& Felecia. (2019). Business intelligence for designing restaurant marketing strategy: A case study. Procedia Computer Science, 161, 615-622. https://doi.org/10.1016/j. procs.2019.11.164

Hu, H. H., Kandampully, J., \& Juwaheer, D. D. (2009). Relationships and impacts of service quality, perceived value, customer satisfaction, and image: An empirical study. Service Industries Journal, 29(2), 111-125. https://doi.org/10.1080/02642060802292932

Kowalkowski, C., Gebauer, H., \& Oliva, R. (2017). Service growth in product firms: Past, present, and future. Industrial Marketing Management, 60, 82-88. https://doi.org/10.1016/j.indmarman.2016.10.015

Kumar, V., \& RRK, S. (2017). An empirical investigation of critical success factors influencing the successful TQM implementation for firms with different strategic orientation. International Journal of Quality \& Reliability Management, 34(9), 1-5.

Larke, R., Kilgour, M., \& O’Connor, H. (2018). Build touchpoints and they will come: transitioning to omnichannel retailing. International Journal of Physical Distribution and Logistics Management, 48(4), 465-483. https://doi.org/10.1108/IJPDLM-09-2016-0276

Madden, T. J., Fehle, F., \& Fournier, S. (2006). Brands matter: An empirical demonstration of the creation of shareholder value through branding. Journal of the Academy of Marketing Science, 34(2), 224-235. https://doi.org/10.1177/0092070305283356

Marin Carrillo, M. B., Marin Carrillo, G. M., \& Jimenez Castillo, D. J. (2004). Análisis de las fuerzas competitivas del sector agrícola de Almería. Boletín Económico de ICE, Información Comercial Española, 2798, 47-60.

Martínez, J. A., \& Martínez, L. (2009). La calidad percibida en servicios deportivos; mapas conceptuales de marca. Revista Internacional de Medicina y Ciencias de La Actividad Fisica y Del Deporte, 9(35), 232-253.

Matthyssens, P. (2019). Reconceptualizing value innovation for Industry 4.0 and the Industrial Internet of Things. Journal of Business and Industrial Marketing, 34(6), 1203-1209. https://doi. org/10.1108/JBIM-11-2018-0348

Milichovsky, F., \& Simberova, I. (2015). Marketing Effectiveness: Metrics for Effective Strategic Marketing.: University of Warwick eResources. 26(2), 211-219. http://0-eds.a.ebscohost.com. pugwash.lib.warwick.ac.uk/eds/pdfviewer/pdfviewer?sid=5fc4b05e-5741-4430-9f05-6b4d$294 \mathrm{f0eb} 8 \% 40$ sessionmgr4008\&vid=1\&hid=4113 
Molina-Morales, F., Martínez-Fernández, T. M., \& Vanina Jasmine, T. (2011). The Dark Side of Trust: The Benefits, Costs and Optimal Levels of Trust for Innovation Performance. Long Range Planning, 44(2), 118-133. https://doi.org/10.1016/j.lrp.2011.01.001

MondayBlog. (2020). Una guía completa para la gestión de marketing. Obtenido de. https://monday. com/blog/es/marketing-es/marketing-management/

Mullins, R. R., \& Panagopoulos, N. G. (2019). Understanding the theory and practice of team selling: An introduction to the special section and recommendations on advancing sales team research. Industrial Marketing Management, 77(xxxx), 1-3. https://doi.org/10.1016/j.indmarman.2018.03.001

Naranjo-Africano, G., \& Giraldo, M. (2020). Corporate Entrepreneurship e innovación: un enlace de dos vías. Revista Venezolana de Gerencia, 25(3). https://doi.org/10.37960/rvg.v25i3.33387

Nowlin, E. L., Walker, D., \& Anaza, N. A. (2018). How does salesperson connectedness impact performance? It depends upon the level of internal volatility. Industrial Marketing Management, 68(January), 106-113. https://doi.org/10.1016/j.indmarman.2017.10.007

Park, C. W., MacInnis, D. J., Priester, J., Eisingerich, A. B., \& Iacobucci, D. (2010). Brand attachment and brand attitude strength. Journal of Marketing, 74(6), 1-17.

Patiño, J. D., Ruiz Ariza, A., \& Pitre-Redondo, R. (2018). El emprendimiento en Colombia, una respuesta a los retos de competitividad y desarrollo sostenible. Espacios, 39(14).

Piercy, N. F., Harris, L. C., Peters, L. D., \& Lane, N. (1997). Marketing management, market strategy and strategic management: Domain realignment and redefinition. Journal of Strategic Marketing, 5(1), 50-62. https://doi.org/10.1080/096525497346866

Pozo, H., \& Tachizawa, T. (2018). Marketing and social responsibility: an exploratory study of local tourism. Tourism \& Management Studies, 14(4), 39-49. https://doi.org/10.18089/ tms.2018.14404

Qazi, A. A., Shaikh, A. R., Shahzad, F., \& Sattar, M. M. (2019). Locate the warehouse: super distribution services. Emerald Emerging Markets Case Studies, 9(2), 1-17. https://doi.org/10.1108/ EEMCS-03-2019-0059

Ramanathan, R., Ramanathan, U., \& Zhang, Y. (2016). Linking operations, marketing and environmental capabilities and diversification to hotel performance: A data envelopment analysis approach. International Journal of Production Economics, 176, 111-122. https://doi.org/10.1016/j.ijpe.2016.03.010

Ramirez Angulo, J., Duque Oliva, E., \& Rodriguez Romero, C. A. (2013). Lealtad de marca : antecedentes y perspectivas de investigación Brand loyalty : Background and perspectives for research. Universidad Y Empres, 24, 141-163.

Reddy, S. K., \& Reinartz, W. (2017). Digital Transformation and Value Creation: Sea Change Ahead. GfK Marketing Intelligence Review, 9(1), 10-17. https://doi.org/10.1515/gfkmir-2017-0002

Rojas De Francisco, L., Bejarano Botero, L. M., \& Marín Valencia, C. F. (2016). Análisis de las estructuras de gestión del servicio en empresas del sector de servicios. AD-Minister, 29, 121146. https://doi.org/10.17230/ad-minister.29.6

Romo Morales, G., \& Márquez de León, E. (2014). Gestión estratégica vs. Análisis estratégico. Una discusión conceptual a partir del estudio de caso de una institución de educación superior. Pensamiento \& gestión, (36), 267-290.

Sainidis, E., Robson, A., \& Heron, G. (2019). Environmental turbulence and the role of business func- 
tions in the manufacturing strategy debate: The case ofUK-based SMEs and the Great Recession. Journal of General Management, 44(4), 190-208. https://doi.org/10.1177/0306307019832498

Sam, A. F. (2017). The Effects of Marketing Mix (4P) on Companies' Profitability: A Case Study of Automotive Industry in France. Journal of Research in Marketing, 8(1), 636. https://doi. org/10.17722/jorm.v8i1.207

Sikora, D. M., Thompson, K. W., Russell, Z. A., \& Ferris, G. R. (2016). Reimagining overqualified human resources to promote organizational effectiveness and competitive advantage. Journal of Organizational Effectiveness: People and Performance, 3(1), 23-42. https://doi. org/10.1108/joepp-03-2015-0012

Sukier, H., Hernández, L., Portillo, R., Valleospino, A., García, M., \& García, J. (2018). Marketing estratégico: una mirada desde el contexto de la empresa familiar. Espacios, 39(44), 9.

Vesga R., J. J. (2013). Cultura organizacional y sistemas de gestión de la calidad: una relación clave en la gestión de las instituciones de educación superior. (Spanish). Revista Científica Guillermo de Ockham, 11(2), 89-100.

Vieira, V. A., da Silva, J. D., \& Gabler, C. (2019). Overcoming over-identification: The power of organizational prestige in optimizing sales performance. Marketing Intelligence and Planning, 37(3), 258-270. https://doi.org/10.1108/MIP-07-2018-0261

Weiss, M., Schneider, D., \& Lebid, J. (2015). Unfolding the concept of a TMT-diversification strategy fit. Team Performance Management: An International Journal, 21(3), 138-154. https://doi. org/10.1108/TPM-03-2014-0028

Wu, M., Zhang, L., Imran, M., Lu, J., \& Hu, X. (2018). Conflict coping strategy evolution of top management team members in China's family enterprises. Chinese Management Studies, 12(2), 246-267. https://doi.org/10.1108/CMS-08-2017-0227 